WUE-ITP-96-009

hep-ph/9606291

\title{
Neutralino mass bounds at the upgraded LEP collider
}

\author{
F. Franke', S. Hesselbach "] \\ Institut für Theoretische Physik, Universität Würzburg \\ D-97074 Würzburg, Germany
}

\begin{abstract}
Assuming that no supersymmetric signature will be found at the upgraded LEP collider we derive lower bounds on the masses of the four neutralinos $\tilde{\chi}_{i}^{0}$ in the Minimal Supersymmetric Standard Model (MSSM). We consider the recently published results from the search for the light chargino $\tilde{\chi}_{1}^{ \pm}$and the next-to-lightest neutralino $\tilde{\chi}_{2}^{0}$ at LEP1.5 and study the consequences of possible future lower $\tilde{\chi}_{1}^{ \pm}$and $\tilde{\chi}_{2}^{0}$ mass limits between 65 and $95 \mathrm{GeV}$. For a chargino mass bound of $66.8 \mathrm{GeV}$ at LEP1.5, a massless neutralino is not excluded for $\tan \beta<1.2$. If either $\tan \beta>2.3$ or the gluino mass $m_{\tilde{g}}>160 \mathrm{GeV}$, we find $m_{\tilde{\chi}_{1}^{0}}>28 \mathrm{GeV}$. A possible chargino bound $m_{\tilde{\chi}_{1}^{ \pm}}>95 \mathrm{GeV}$ at LEP2 would raise this bound to $31 \mathrm{GeV}$ (for all $\tan \beta$ ) or $44 \mathrm{GeV}(\tan \beta>2)$.
\end{abstract}

May 1996

*email: fabian@physik.uni-wuerzburg.de

$\dagger$ †email: hesselb@physik.uni-wuerzburg.de 


\section{Introduction}

The upgrading of the LEP collider which has already started with an increase of the available center-of-mass energy from $\sqrt{s} \approx m_{Z}$ (LEP1) to $136 \mathrm{GeV}$ (LEP1.5) and will continue up to $\sqrt{s} \approx 190 \mathrm{GeV}$ (LEP2) in 1997 opens fascinating opportunities for precision tests of the standard model (SM) as well as for detecting first signatures of new physics. It is widely acknowledged that supersymmetry (SUSY) [1] is the most likely theory beyond the SM. Therefore the search for supersymmetric particles plays a fundamental role at the present and future high-energy colliders and also in the program of the upgraded LEP [2]. Until now, however, no direct evidence for SUSY has been found. Therefore the experiments at LEP and TEVATRON resulted in lower mass limits for SUSY particles. At the upgraded LEP, one expects either the spectacular identification of a SUSY particle or the lower mass bounds will increase. Under the assumption that the production of SUSY particles is kinematically allowed, there exist several channels for the detection of a supersymmetric signature. Among the most promising processes is the pair production of neutralinos or charginos, the mass eigenstates of the fermionic partners of the gauge and Higgs bosons.

In the present paper we use the Minimal Supersymmetric Standard Model (MSSM) [3] as framework for the calculation of the neutralino mass reach to be probably covered at the upgraded LEP collider. In the MSSM one of the charged superpartners is expected to be the first SUSY particle to be detected or excluded up to a mass of $\sim \sqrt{s} / 2$. Mainly the light chargino $\tilde{\chi}_{1}^{ \pm}$and a light scalar top quark $\tilde{t}_{1}$ are discussed to be the lightest visible supersymmetric particle [2]. In order to estimate the chances for a supersymmetric signature one has to analyze carefully the possible decay channels. Due to R-parity conservation in the MSSM, all decay products contain the invisible lightest supersymmetric particle (LSP) which is assumed to be the lightest neutralino $\tilde{\chi}_{1}^{0}$. In the case of stop and chargino production, there are also charged quarks or leptons in the final state which could lead to a clear signature identifying or ruling out the respective SUSY particle. The production cross sections for stops [4] and charginos [5] and their branching ratios as a function of the supersymmetric parameters are well-known for LEP2 energies and form the theoretical basis for the experimental search at LEP.

Therefore the first results of the LEP1.5 run with $\sqrt{s}=136 \mathrm{GeV}$ [6, 7, 8, 9, 10] all contain a discussion of the chargino search. Since no supersymmetric signature was found, the LEP collaborations have reported a lower limit on the mass of the light chargino $m_{\tilde{\chi}_{1}^{ \pm}}>65 \mathrm{GeV}$ with some dependence on the chargino mixing, the sneutrino mass and the mass difference to the LSP. The DELPHI collaboration [7] has set a preliminary limit

of $m_{\tilde{\chi}_{1}^{ \pm}}>66.8 \mathrm{GeV}$ for $m_{\tilde{\chi}_{1}^{ \pm}}-m_{\tilde{\chi}_{1}^{0}}>10 \mathrm{GeV}$ and $m_{\tilde{\chi}_{1}^{ \pm}}>63.8 \mathrm{GeV}$ for $m_{\tilde{\chi}_{1}^{ \pm}}-m_{\tilde{\chi}_{1}^{0}}=5 \mathrm{GeV}$ and a sneutrino mass of $1 \mathrm{TeV}$, while ALEPH 8 found a lower mass bound of $67.8 \mathrm{GeV}$ for gaugino-like charginos and the sneutrino heavier than $200 \mathrm{GeV}$, or $65 \mathrm{GeV}$ for a higgsinolike chargino when the mass difference to the LSP is larger than $10 \mathrm{GeV}$. Finally OPAL [10] derived lower chargino mass bounds between 60.7 (58.7) GeV for the smallest possible universal scalar mass $m_{0}$ and 65.4 (65.6) $\mathrm{GeV}$ for $m_{0}>1 \mathrm{TeV}$ and $\tan \beta=1.5$ (35), again with the mass difference condition $m_{\tilde{\chi}_{1}^{ \pm}}-m_{\tilde{\chi}_{1}^{0}}>10 \mathrm{GeV}$.

Another candidate for the lightest visible supersymmetric particle is the second lightest neutralino $\tilde{\chi}_{2}^{0}$ which can be identified by its decay into the LSP. The dominant decay 
channels, however, significantly depend on the neutralino mixing and vary within different regions of the parameter space [11]. With the results of the LEP1.5 run, the ALEPH and OPAL collaborations have set limits on the cross sections $\sigma\left(e^{+} e^{-} \rightarrow \tilde{\chi}_{1}^{0} \tilde{\chi}_{2}^{0}\right)$ as a function of the neutralino masses [8, 10]. For a higgsino-like $\tilde{\chi}_{2}^{0}$ and $m_{\tilde{\chi}_{2}^{0}}-m_{\tilde{\chi}_{1}^{0}}>10 \mathrm{GeV}$ ALEPH found a lower mass bound for the second lightest neutralino of $m_{\tilde{\chi}_{2}^{0}}>69 \mathrm{GeV}$. The most detailed neutralino mass bounds at LEP1.5 are derived by the OPAL collaboration. Their $\tilde{\chi}_{1}^{0}\left(\tilde{\chi}_{2}^{0}\right)$ bounds range from $12.0(45.3) \mathrm{GeV}$ for a minimal universal scalar mass $m_{0}$ and $\tan \beta=1.5$ to $35.2(67.5) \mathrm{GeV}$ for $m_{0}=1 \mathrm{TeV}$ and $\tan \beta=35$ with a mass difference between the two light neutralinos larger than $10 \mathrm{GeV}$. But also OPAL does not yet study precisely the dependence on $\tan \beta$.

In this paper, however, we consider general neutralino mixing, scan over a wide theoretically acceptable parameter range and do not impose any restrictions on the mass difference to the LSP or mixing types. We mainly pursue two aims: First we want to analyze the lower mass bounds for all four neutralinos derived from the LEP1.5 results. Here, we also consider the effect of the TEVATRON constraints on the gluino mass bounding the SUSY parameter $M$. Second, we want to study the development of the bounds on the way to LEP2 if no neutralino or chargino is found. Therefore we consider possible future chargino mass bounds up to $95 \mathrm{GeV}$ and also the consequences of such a bound for the second lightest neutralino. Similar studies for LEP1 were performed e.g. in [12].

In our analysis we have to take into account that the masses and mixings of charginos [5] and neutralinos [13] are strongly correlated. Both mixing matrices depend on the same parameters, namely the $S U(2)$ and $U(1)$ gaugino masses $M^{\prime}$ and $M$, which are connected by the usual GUT relation $M^{\prime} / M=5 / 3 \tan ^{2} \theta_{W}$, the $\mu$ parameter in the superpotential and the ratio of the vacuum expectation values of the Higgs doublets $\tan \beta=v_{2} / v_{1}$. Therefore new chargino mass bounds also result in lower mass bounds for the neutralinos even without considering experimental constraints in the neutralino sector. In fact it turns out that the constraints from negative chargino search represent the by far stronger criterium for the exclusion of a parameter region. Only a small domain is additionally excluded by neutralino constraints. In view of the further increase of the LEP energy towards LEP2 we therefore study the consequences for the neutralino mass bounds as a function of the chargino bounds. In a second step we also include in our analysis possible new lower limits on the mass of the second lightest neutralino up to $95 \mathrm{GeV}$ which may arise by the neutralino search at LEP2. Our results make it possible to determine neutralino mass bounds immediately when new chargino bounds are announced from a LEP run with increased energy.

The paper begins with a short analysis of the parameter domain excluded by LEP1.5 in combination with the neutralino and chargino mass contour lines in the $(M, \mu)$ plane needed for the interpretation of the following figures. Then we present lower mass limits for the four neutralinos as a function of $\tan \beta$ and of prospective new mass bounds for the light chargino and the second lightest neutralino. Finally we explicitly give the neutralino mass limits for the cases of $m_{\tilde{\chi}_{1}^{ \pm}}>66.8 \mathrm{GeV}$ (LEP1.5) and $m_{\tilde{\chi}_{1}^{ \pm}}>95 \mathrm{GeV}$ (LEP2). All results are compared with the corresponding neutralino bounds from LEP1 [14]. 


\section{Parameter constraints}

In this paper, we start with the conservative LEP1.5 chargino bound of the DELPHI collaboration [7]

$$
m_{\tilde{\chi}_{1}^{ \pm}} \geq 66.8 \mathrm{GeV}
$$

and discuss the case that this bound may be raised up to $95 \mathrm{GeV}$ by LEP2. Furthermore we consider the neutralino constraints from LEP1 [14] and LEP1.5 [8]. In particular, we use

1. the limit on the total $Z$ width

$$
\Delta \Gamma_{Z} \leq 23.1 \mathrm{MeV},
$$

where

$$
\Delta \Gamma_{Z}=\Gamma\left(Z \rightarrow \tilde{\chi}_{i}^{0} \tilde{\chi}_{j}^{0}\right)+\Gamma\left(Z \rightarrow \tilde{\chi}_{k}^{ \pm} \tilde{\chi}_{l}^{\mp}\right), \quad i, j=1, \ldots 4 ; k, l=1,2 ;
$$

2. the limit on the invisible $Z$ width

$$
\Delta \Gamma_{\mathrm{inv}} \leq 8.4 \mathrm{MeV}
$$

where

$$
\Delta \Gamma_{\mathrm{inv}}=\Gamma\left(Z \rightarrow \tilde{\chi}_{1}^{0} \tilde{\chi}_{1}^{0}\right) ;
$$

3. the limits from direct neutralino search at the $Z$-resonance

$$
\begin{array}{ll}
B R\left(Z \rightarrow \tilde{\chi}_{1}^{0} \tilde{\chi}_{j}^{0}\right)<2 \times 10^{-5} & j=2, \ldots 4, \\
B R\left(Z \rightarrow \tilde{\chi}_{i}^{0} \tilde{\chi}_{j}^{0}\right)<5 \times 10^{-5} & i, j=2, \ldots 4 ;
\end{array}
$$

4. the limit on the cross section from direct neutralino search at LEP1.5

$$
\sigma\left(e^{+} e^{-} \rightarrow \tilde{\chi}_{1}^{0} \tilde{\chi}_{2}^{0}\right)<5,3,1.8,1.4 \mathrm{pb}
$$

as a function of the masses of the lightest and next-to-lightest neutralino as given in ref. [8]. In our calculations, we apply this limit to all neutralino pair production channels with at least one visible neutralino.

In fact one has to take into account the results from both LEP1 and LEP1.5 since the LEP1.5 bounds of eqs. (1) and (8) do not totally cover the LEP1 constraints of eqs. (2) - (7).

We will discuss the excluded parameter space and the neutralino mass bounds in detail for the lowest possible value $\tan \beta=1$, a small value of $\tan \beta=2$ and a larger $\tan \beta=10$, but we will also analyze the dependence of the neutralino mass bounds on $\tan \beta$ with the LEP1.5 and the possible LEP2 results. Generally, the computed mass bounds do not significantly change for a further increased $\tan \beta>10$. With $\tan \beta=1$ we explicitly want to study the light neutralino window which allowed massless neutralinos for $\tan \beta<1.7$ at LEP1. 
The excluded parameter domain in the $(M, \mu)$ plane for $\tan \beta=1,2,10$ from the $Z$ width measurements and the direct neutralino and chargino search at LEP1 and LEP1.5 is shown in Fig. 1. For all our calculations of mass bounds in this paper we consider the SUSY parameter range $0<M<400 \mathrm{GeV}$ and $-500 \mathrm{GeV}<\mu<500 \mathrm{GeV}$ of Fig. 1 . The parameter regions that are excluded by the negative chargino search at LEP1.5 and that may be covered at LEP2 are marked by the contour lines for a chargino mass of $66.8 \mathrm{GeV}$ and $95 \mathrm{GeV}$, respectively. The values of $\tan \beta$ in Fig. 1 represent the three different cases how this parameter domain is extended by direct neutralino searches at LEP1 and LEP1.5. For $\tan \beta=1$ direct neutralino searches at both LEP energies lead to exclusively excluded parameter regions additionally to the chargino search (Fig. 1a). Nevertheless massless neutralinos remain allowed for small parameters $|\mu|$ and $M$. This light neutralino window depending on $\tan \beta$ will be discussed in Sec. 3. The situation is different for increasing $\tan \beta$ where for $\tan \beta=2$ a small parameter region with small negative $\mu$ is excluded only by the direct neutralino search at LEP1, while the LEP1.5 limit of eq. (8) does not extend the parameter domain excluded by chargino search (Fig. 1b). For the large value $\tan \beta=10$ (Fig. 1c), the chargino bound alone determines the excluded parameter space, since it is stronger than the neutralino bounds from both LEP1 and LEP1.5 over the whole $(M, \mu)$ plane. Therefore one may use for the calculation of the lower neutralino mass bounds only the chargino bounds if $\tan \beta>2$, while for smaller $\tan \beta$ also the results from the direct neutralino search at LEP1 and LEP1.5 have to be included. A LEP2 chargino bound of $95 \mathrm{GeV}$, however, would definitely improve all parameter constraints from neutralino search at LEP1 and LEP1.5 for all values of $\tan \beta$.

In the following section we will discuss the neutralino masses which are compatible with the allowed parameter domains. The contour lines for the mass of the lightest neutralino shown in Fig. 1 may help to explain the lower mass bound for the lightest neutralino. For LEP1.5 and thereafter, this bound is mainly determined by the chargino mass limit with the exceptions described above.

\section{$3 \quad$ Neutralino mass bounds}

In Fig. 2 the lower neutralino mass bounds are shown as a function of $\tan \beta$ for the new chargino bound $m_{\tilde{\chi}_{1}^{ \pm}}>66.8 \mathrm{GeV}$ of LEP1.5 (Fig. 2a) and for a prospective bound $m_{\tilde{\chi}_{1}^{ \pm}}>95 \mathrm{GeV}$ after LEP2 (Fig. 2b). Note that we do not consider any assumptions on the mixing type or on the mass difference between a visible neutralino and the LSP. Therefore our bound on $\tilde{\chi}_{2}^{0}$ is significantly lower than the LEP1.5 bound published by ALEPH [8]. Generally, the experimental results from LEP1.5 raise the neutralino mass limits by $5-10 \mathrm{GeV}$ compared to the bounds from LEP1 [14]. But even LEP1.5 does not totally exclude a massless neutralino for small $\tan \beta<1.2$, while it was allowed up to $\tan \beta<1.7$ at LEP1. One would need a lower chargino mass bound of about $78 \mathrm{GeV}$ in order to rule out a massless neutralino at LEP for all $\tan \beta$. If no chargino will be found at LEP2, a lower bound $m_{\tilde{\chi}_{1}^{0}}>31 \mathrm{GeV}$ can be expected independently of $\tan \beta$.

A lower bound on the gluino mass $m_{\tilde{g}}$, however, can raise these LEP1.5 neutralino bounds. The CDF gluino mass limits significantly depend on the squark mass [15]. As- 
suming a heavy squark $m_{\tilde{q}}>400 \mathrm{GeV}$, the moderate bound

$$
m_{\tilde{g}}>160 \mathrm{GeV}
$$

restricts the parameter $M$ using the GUT relation

$$
M=\frac{\alpha_{2}}{\alpha_{3}} m_{\tilde{g}} \approx 0.3 m_{\tilde{g}}
$$

where $\alpha_{2}$ and $\alpha_{3}$ are the gauge coupling constants of the $S U(2)$ and $S U(3)$ gauge groups, respectively. We show in Fig. 2a also the lower neutralino mass bounds taking into account this restriction

$$
M>50 \mathrm{GeV} .
$$

In this case there exists a lower neutralino mass bound of $28 \mathrm{GeV}$ for all values of $\tan \beta$, a massless neutralino can be ruled out. Generally, the LEP1.5 mass bounds for all four neutralinos are raised compared to LEP1 for small $\tan \beta$ with this gluino mass limit, while it has no effect for $\tan \beta>2.5$.

In order to have also any influence on the prospective neutralino bounds at LEP2, a gluino mass limit of at least $300 \mathrm{GeV}$ is necessary (see Fig. 1). Therefore we do not discuss such a limit in Fig. 2b but study in Figs. $2 \mathrm{~b}$ and 3 the impact of an experimental lower bound for the lightest visible neutralino $\tilde{\chi}_{2}^{0}$ of the same size as the chargino bound. This $\tilde{\chi}_{2}^{0}$ bound serves as a rough estimate for the possible results from direct neutralino search at LEP2 which cannot yet be determined in detail.

Just with the chargino limit $m_{\tilde{\chi}_{1}^{ \pm}}>95 \mathrm{GeV}$ (solid lines), the lightest visible neutralino $\tilde{\chi}_{2}^{0}$ is heavier than $54 \mathrm{GeV}(\tan \beta=1)$ or $95 \mathrm{GeV}(\tan \beta=10)$. Now by supposition this bound on $\tilde{\chi}_{2}^{0}$ is always $95 \mathrm{GeV}$ in Fig. $2 \mathrm{~b}$ (dashed lines). Then the mass bound on $\tilde{\chi}_{4}^{0}$ is significantly higher for small $\tan \beta<8$ by as much as $25 \mathrm{GeV}$. The bounds on $\tilde{\chi}_{1}^{0}$ and $\tilde{\chi}_{3}^{0}$ are raised by about $3-5 \mathrm{GeV}$ only for small $\tan \beta<1.5$, for larger $\tan \beta$ they remain practically unaffected by the additional $\tilde{\chi}_{2}^{0}$ bound.

Now we give an outlook on the possible increase of the neutralino mass limits when new chargino mass bounds and additional bounds on the lightest visible neutralino arise during the further upgrading of LEP. In Fig. 3 the neutralino bounds are depicted as a function of the chargino limit for the two values $\tan \beta=2,10$. For $\tan \beta=2$ the $\tilde{\chi}_{1}^{0}$ $\left(\tilde{\chi}_{2}^{0}\right)$ mass limit increases from 25 (48) $\mathrm{GeV}$ with $m_{\tilde{\chi}_{1}^{ \pm}}>65 \mathrm{GeV}$ to 44 (77) $\mathrm{GeV}$ with $m_{\tilde{\chi}_{1}^{ \pm}}>95 \mathrm{GeV}$. Larger values of $\tan \beta$ lead to a further increase of the lower mass limits between $8 \mathrm{GeV}$ for the lightest neutralino and $60 \mathrm{GeV}$ for $\tilde{\chi}_{4}^{0}$.

The dashed lines in Fig. 3 show the lower limits on the masses of $\tilde{\chi}_{1,3,4}^{0}$ if additionally to a lower chargino mass bound also the same mass limit for the lightest visible neutralino $\tilde{\chi}_{2}^{0}$ is derived by direct neutralino search. Here for small $\tan \beta$ a similar increase of the $\tilde{\chi}_{2}^{0}$ and $\tilde{\chi}_{4}^{0}$ bounds occurs as already discussed in Fig. 2, while the other neutralino mass bounds for $\tan \beta=2$ and all bounds for $\tan \beta=10$ remain nearly unaffected. Thus for large values of $\tan \beta$ it is the chargino mass limit alone that determines the lower neutralino mass limits at LEP2.

The neutralino bounds of Figs. 2 and 3 are summarized in Table 1 which can easily be compared with the results of LEP1 (for the most recent analysis of the LEP1 data see Table 3 in [14], earlier results can be found in [16]). Except for the heaviest neutralino, 
there is a significant raise of the lower neutralino mass limits for $\tan \beta>1.2$. Already now after LEP1.5 the lightest neutralino $\tilde{\chi}_{1}^{0}$ must be heavier than $25 \mathrm{GeV}$ compared to $20 \mathrm{GeV}$ after LEP1 if $\tan \beta>2$. If LEP2 sets a lower chargino mass bound of $95 \mathrm{GeV}$, the $\tilde{\chi}_{1}^{0}$ mass is larger than at least $31 \mathrm{GeV}($ all $\tan \beta)$ or $44 \mathrm{GeV}(\tan \beta>2)$ and a massless neutralino is excluded. Experimental results from direct neutralino search at LEP2 may further improve these bounds. Including the CDF bound on the gluino mass $m_{\tilde{g}}>160 \mathrm{GeV}$, we obtain with the LEP1.5 results lower mass bounds of 28 and $35 \mathrm{GeV}$ for the lightest and next-to-lightest neutralino, respectively, independently of the value for $\tan \beta$.

\section{Conclusion}

We have derived lower limits on the masses of the four neutralinos if the experiments at the upgraded LEP collider yield new chargino mass bounds between 65 and $95 \mathrm{GeV}$. The preliminary limit $m_{\tilde{\chi}_{1}^{ \pm}}>66.8 \mathrm{GeV}$ announced by the LEP collaborations does not yet exclude massless neutralinos for $\tan \beta<1.2$. A future chargino mass bound $m_{\tilde{\chi}_{1}^{ \pm}}>$ $78 \mathrm{GeV}$, however, would definitely rule out the existence of massless neutralinos for all values of $\tan \beta$.

For $\tan \beta>2$, the lower limit on the mass of the lightest neutralino is found to be $25 \mathrm{GeV}$ for the new LEP1.5 chargino mass limit. With the additional constraint on the gluino mass $m_{\tilde{g}}>160 \mathrm{GeV}(M>50 \mathrm{GeV})$ suggested by the TEVATRON results this bound is raised to $m_{\tilde{\chi}_{1}^{0}}>28 \mathrm{GeV}$ for all values of $\tan \beta$. If no chargino is discovered at LEP2 and a chargino mass bound $m_{\tilde{\chi}_{1}^{ \pm}}>95 \mathrm{GeV}$ is established, one gets lower $\tilde{\chi}_{1}^{0}$ $\left(\tilde{\chi}_{2}^{0}\right)$ limits of 31 (54) GeV for all values of $\tan \beta$. Therefore LEP2 will definitely find or exclude massless neutralinos in the MSSM, which are still allowed after LEP1.5 if the gaugino mass parameter $M$ is not constrained.

\section{Acknowledgements}

We would like to thank H. Fraas and A. Bartl for the careful reading of the manuscript. This work was supported by the Deutsche Forschungsgemeinschaft under contract no. FR 1064/2-1.

\section{References}

[1] Y.A. Gol'fand and E.P Likhtman, JETP Lett. 13 (1971) 323;

D.V. Volkov and V.P. Akulov, Phys. Lett. B 46 (1973) 109;

J. Wess and B. Zumino, Nucl. Phys. B 70 (1974) 39; Phys. Lett. B 49 (1974) 52;

Nucl. Phys. B 78 (1974) 1

[2] G.F. Giudice et al., in "Physics at LEP2", eds. G. Altarelli, T. Sjöstrand and F. Zwirner, CERN 96-01, p. 463

[3] H.E. Haber and G.L. Kane, Phys. Rep. 117 (1985) 75 
[4] A. Bartl, H. Eberl, S. Kraml, M. Majerotto and W. Porod, UWThPh-1996-18, HEPHY-PUB 642/96, hep-ph/9603410, to appear in Z. Phys. C

[5] A. Bartl, H. Fraas, W. Majerotto and B. Mösslacher, Z. Phys. C 55 (1992) 257

[6] L. Rolandi, H. Dijkstra, D. Strickland and G. Wilson, representing the ALEPH, DELPHI, L3 and OPAL collaborations, Joint Seminar on the First Results from LEP1.5, CERN, December 12th, 1995

[7] DELPHI Collaboration, P. Abreu et al., Phys. Lett. B 382 (1996) 323

[8] ALEPH Collaboration, D. Buskulic et al., Phys. Lett. B 373 (1996) 246

[9] L3 Collaboration, M. Acciarri et al., Phys. Lett. B 377 (1996) 289

[10] OPAL Collaboration, G. Alexander et al., Phys. Lett. B 377 (1996) 181

[11] S. Ambrosanio and B. Mele, Phys. Rev. D 52 (1995) 3900; D 53 (1996) 2541

[12] L. Roszkowski, Phys. Lett. B 252 (1990) 471;

M. Drees and X. Tata, Phys. Rev. D 43 (1991) 2971;

H. Baer, X. Tata and J. Woodside, Phys. Rev. D 44 (1991) 207;

K. Hidaka, Phys. Rev. D 44 (1991) 927

[13] A. Bartl, H. Fraas, W. Majerotto and N. Oshimo, Phys. Rev. D 40 (1989) 1594

[14] L3 Collaboration, M. Acciarri et al., Phys. Lett. B 350 (1995) 109

[15] CDF Collaboration, F. Abe et al., Phys. Rev. Lett. 76 (1996) 2006

[16] ALEPH Collaboration, D. Decamp et al., Phys. Lett. B 244 (1990) 541; Phys. Rep. 216 (1992) 253;

DELPHI Collaboration, P. Abreu et al., Phys. Lett. B 247 (1990) 157;

OPAL Collaboration, M.Z. Akrawy et al., Phys. Lett. B 248 (1990) 211 


\begin{tabular}{ccrrrc}
\hline Chargino & Neutralino & \multicolumn{3}{c}{$\tan \beta$} & $m_{\tilde{g}}>160 \mathrm{GeV}$ \\
\cline { 3 - 5 } mass bound & & $>1$ & $>2$ & $>3$ & all $\tan \beta$ \\
\hline 66.8 & $\tilde{\chi}_{1}^{0}$ & 0 & 25 & 31 & 28 \\
& $\tilde{\chi}_{2}^{0}$ & 0 & 48 & 54 & 35 \\
& $\tilde{\chi}_{3}^{0}$ & 75 & 84 & 89 & 83 \\
& $\tilde{\chi}_{4}^{0}$ & 91 & 115 & 128 & 106 \\
\hline 95 & $\tilde{\chi}_{1}^{0}$ & 31 & 44 & 46 & 31 \\
& $\tilde{\chi}_{2}^{0}$ & 54 & 77 & 86 & 54 \\
& $\tilde{\chi}_{3}^{0}$ & 99 & 109 & 113 & 99 \\
& $\tilde{\chi}_{4}^{0}$ & 106 & 137 & 159 & 106 \\
\hline
\end{tabular}

Table 1: Lower neutralino mass limits for two lower chargino mass bounds in GeV. 


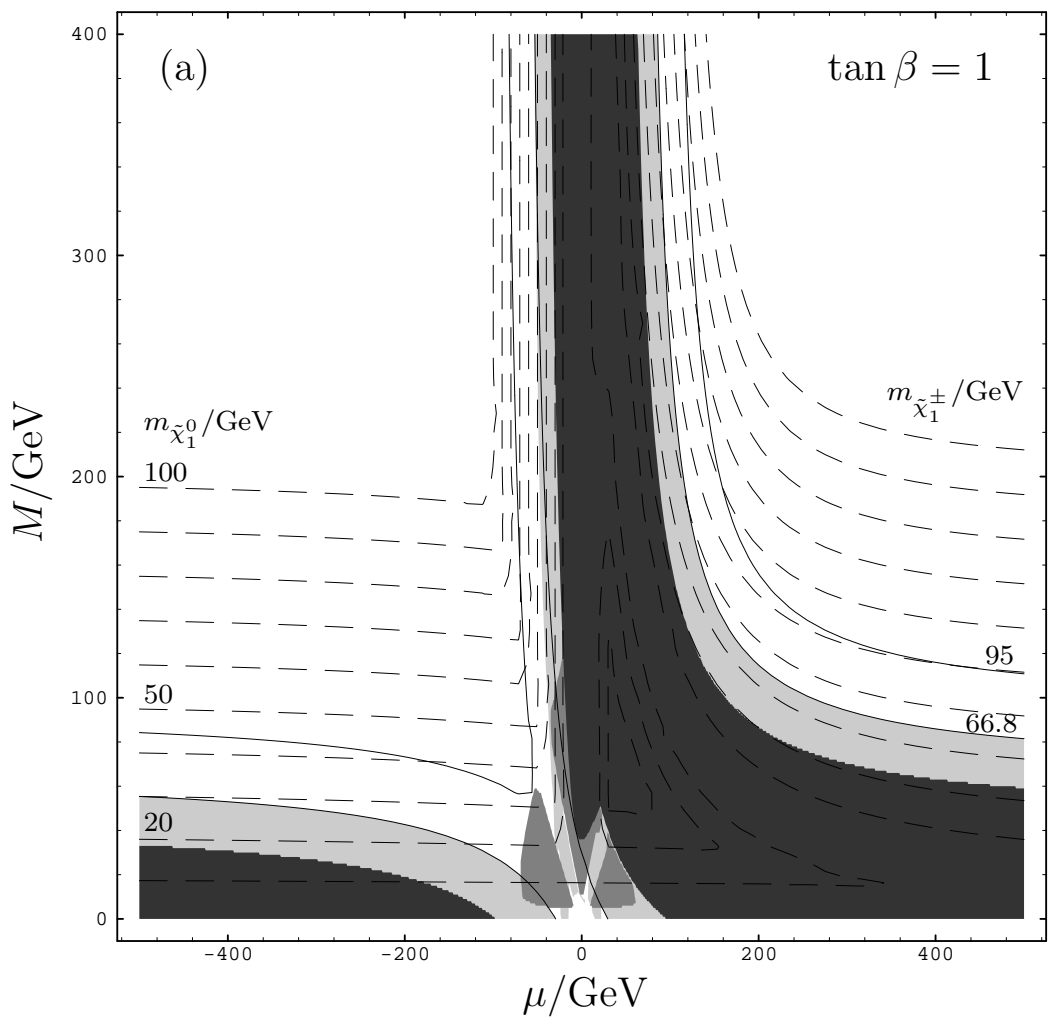

Figure 1a: Contour lines for the mass of the lightest neutralino (dashed; 10, 20, 30, 40, 50, 60, 70, 80, 90 and $100 \mathrm{GeV}$ ) and chargino (solid; 66.8 and $95 \mathrm{GeV}$ ) and the excluded parameter space from LEP1 (dark: $Z$ width measurements, gray: direct neutralino search) and LEP1.5 (bright shaded) in the $(M, \mu)$ plane for $\tan \beta=1$. 


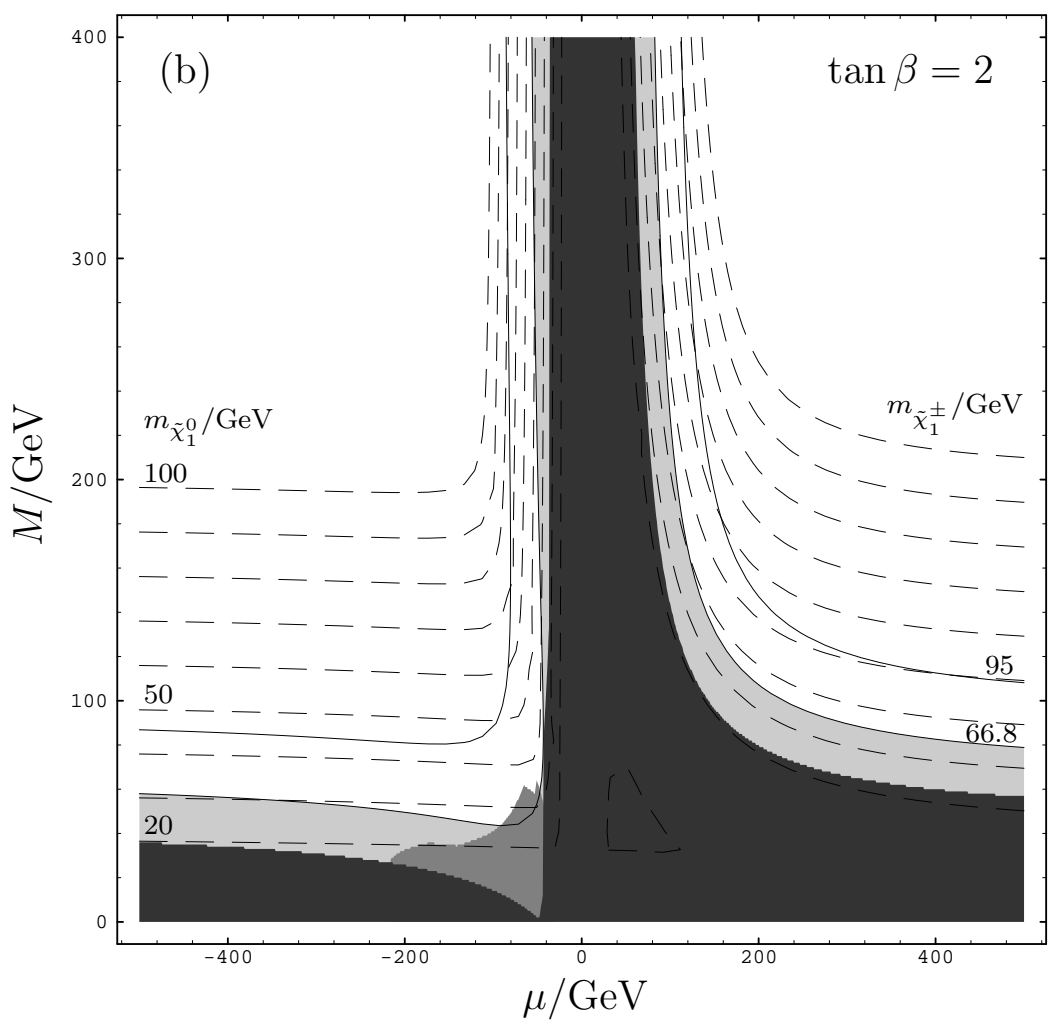

Figure 1b: Contour lines for the mass of the lightest neutralino (dashed; 20, 30, 40, 50, 60, 70, 80, 90 and $100 \mathrm{GeV}$ ) and chargino (solid; 66.8 and $95 \mathrm{GeV}$ ) and the excluded parameter space from LEP1 (dark: $Z$ width measurements, gray: direct neutralino search) and LEP1.5 (bright shaded) in the $(M, \mu)$ plane for $\tan \beta=2$. 


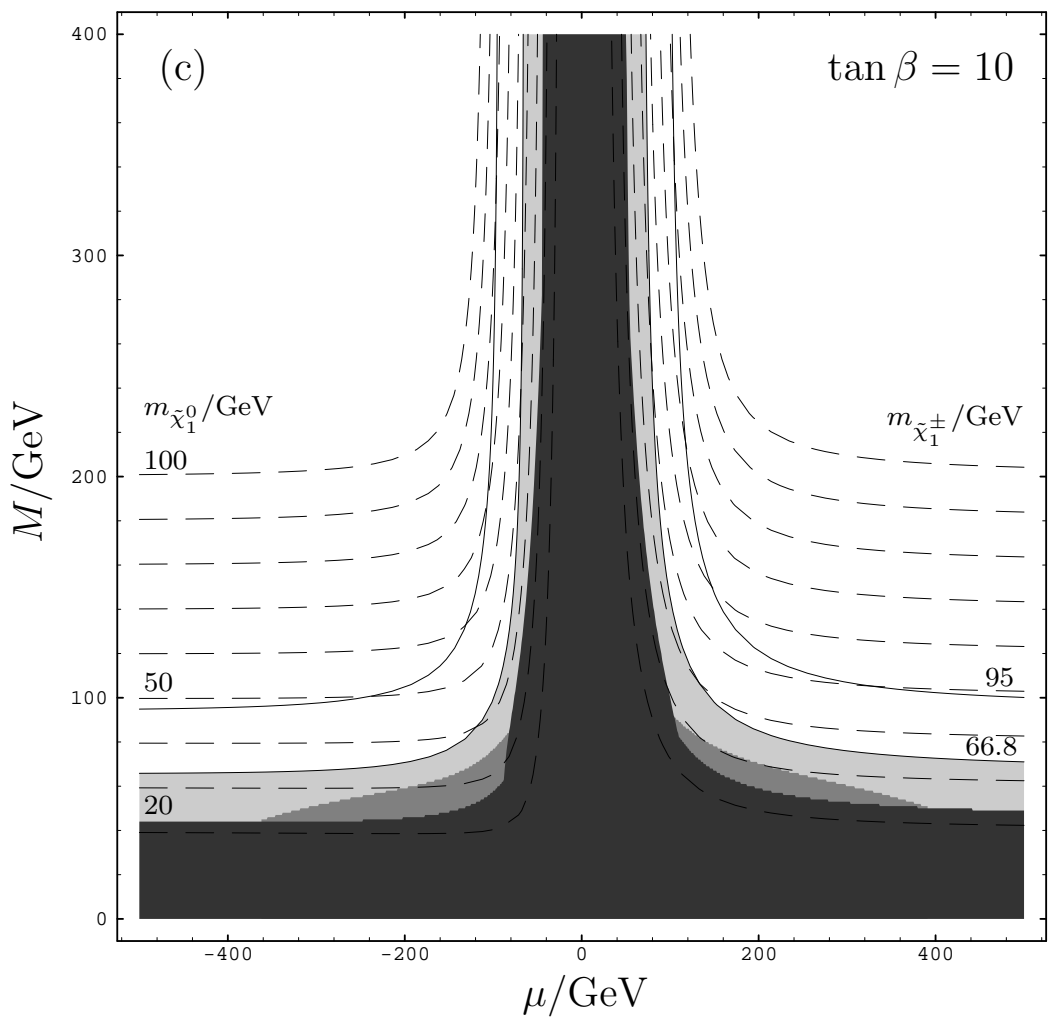

Figure 1c: Contour lines for the mass of the lightest neutralino (dashed; 20, 30, 40, 50, 60, 70, 80, 90 and $100 \mathrm{GeV}$ ) and chargino (solid; 66.8 and $95 \mathrm{GeV}$ ) and the excluded parameter space from LEP1 (dark: $Z$ width measurements, gray: direct neutralino search) and LEP1.5 (bright shaded) in the $(M, \mu)$ plane for $\tan \beta=10$. 

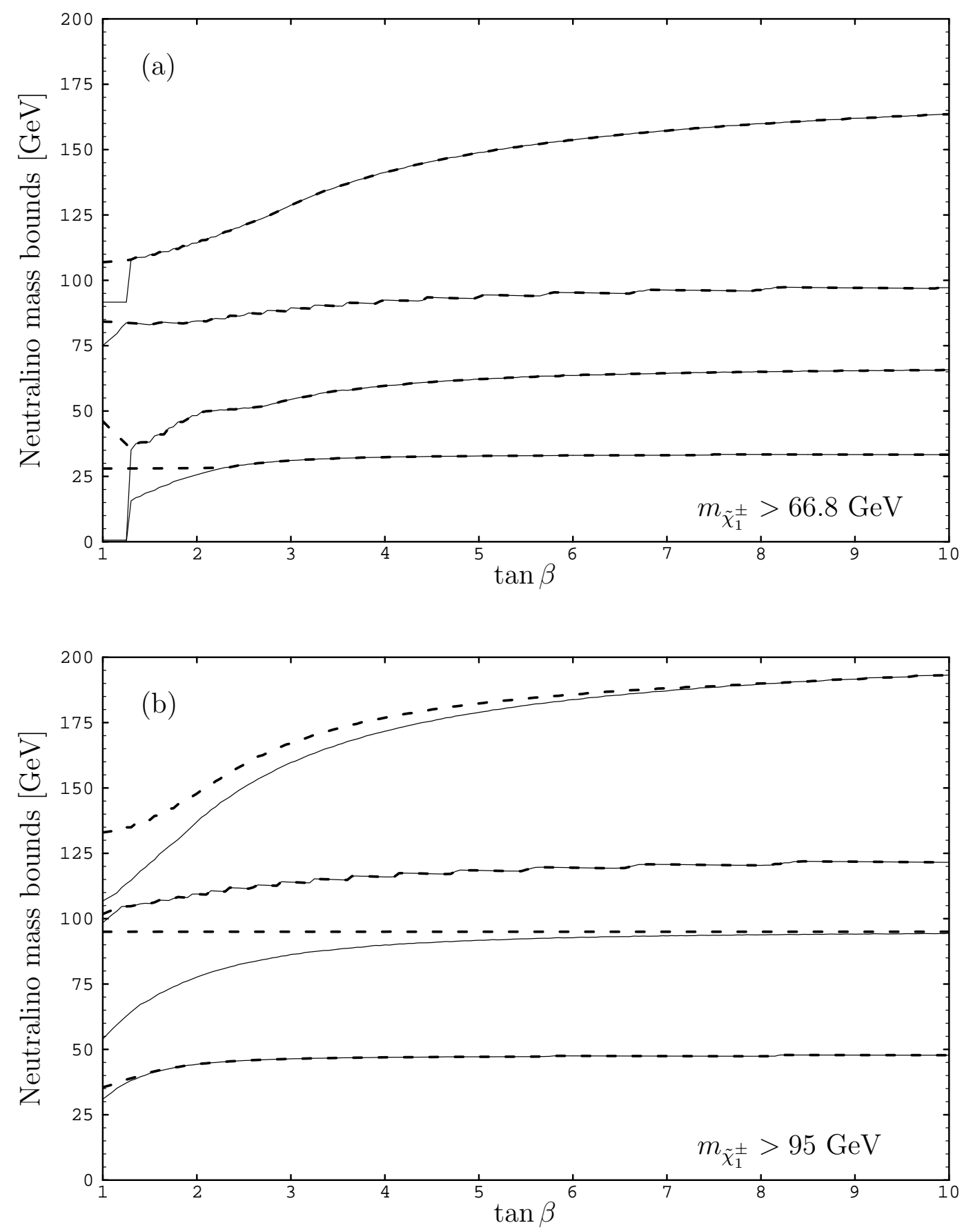

Figure 2: The lower limits on the masses of the four neutralinos as a function of $\tan \beta$ for the chargino mass bounds $m_{\tilde{\chi}_{1}^{ \pm}}>66.8 \mathrm{GeV}$ (a) and $m_{\tilde{\chi}_{1}^{ \pm}}>95 \mathrm{GeV}$ (b). The dashed lines in (a) include the CDF bound $M>50 \mathrm{GeV}$, whereas in (b) they mark the mass limits if additionally the second lightest neutralino is found to be heavier than $95 \mathrm{GeV}$. 

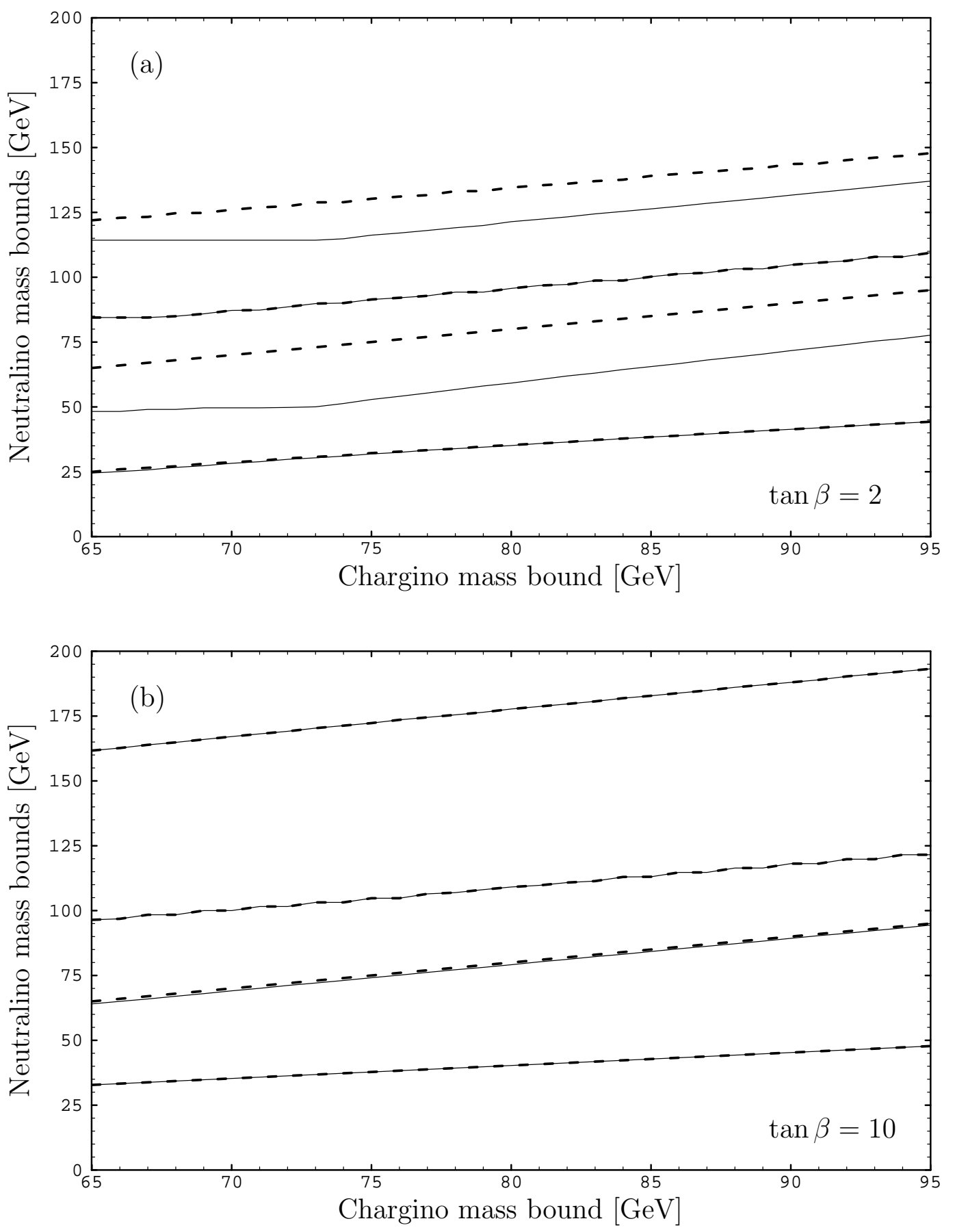

Figure 3: The lower limits on the masses of the four neutralinos as a function of the lower chargino mass bound for $\tan \beta=2$ (a) and $\tan \beta=10$ (b). The dashed lines are valid if there exists the same mass bound for the lightest visible neutralino $\tilde{\chi}_{2}^{0}$. 